\title{
Covid-19 and Its Gastrointestinal Implications
}

\author{
Brian França dos Santos ${ }^{1,3 *}$, Daubernai Bonoso Monteiro Neto ${ }^{2}$ and Lucrécia Helena Loureiro ${ }^{3}$ \\ ${ }^{1}$ Iguaçu University, UNIG, Medicine School, Brazil \\ ${ }^{2}$ Souza Aguiar Municipal Hospital, Brazil \\ ${ }^{3}$ MECSMA/UniFOA, Brazil
}

*Corresponding author: Brian França dos Santos, Iguaçu University, UNIG, Medicine School, Brazil.

To Cite This Article: Brian Franca dos S, Daubernai Bonoso Monteiro N, Lucrécia Helena L. Covid-19 and Its Gastrointestinal Implications. 2020 9(4). AJBSR.MS.ID.001414. DOI: 10.34297/AJBSR.2020.09.001414.

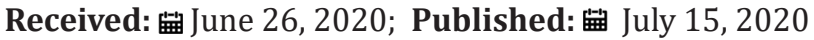

\begin{abstract}
Introduction: Since the 2019 Coronavirus Disease (COVID-19) outbreak in December 2019, several digestive symptoms have been frequently reported in patients infected with the virus. In this study, we aimed to report the case of a patient with COVID-19 with digestive symptoms.

Methods: The information was obtained through a review of the medical records, interview with the patient, photographic record of the diagnostic methods; laboratory tests; Computed tomography of the chest, including RT-PCR for the diagnosis of COVID-19, in addition to a literature review.
\end{abstract}

Results: Digestive symptoms are common in patients with COVID-19.

Conclusions: Physicians should recognize that digestive symptoms, such as diarrhea, are usually among the characteristics of COVID-19 and that the index of suspicion may need to be recognized earlier in patients with digestive symptoms.

\section{Introduction}

First pneumonia of reports etiology unknown was identified in Wuhan, city from Hubei, in Chinese Provence, in 2019 December [1]. On 29 March 2020 more than 700.000 cases were confirmed around the world. This fast dissemination disses with the World Health Organization (WHO) also categorized this world event as a pandemic caused by SARS-COV-2 virus, also known by Coronavirus and Covid-19.

Previously referred as 2019-COV by WHO, the SARS-COV was identified by "International Committee on Taxonomy of Viruses", is a new Betacoronavirus that infect humans. According to their genetic similarity to two others Coronavirus, like SARS, virus that causes severe acute respiratory syndrome, its origin has been attributed by bats [2]. We know a little about SARS origin virus, however, there has been a transition of some kind unknown to humans in seafood Market, located in Wuhan Chinese City, yet. So, is important to show the exact transmission route needs to be clarify [3].

The transmission virus from person to person is by droplets, when the virus is laden by small droplets original nose and mouth infected people, when talking, exhale and sneeze. The infection can also occur when a person touches surfaces or contaminated objects and this way, touch the eyes, nose or mouth. Scientific reports show that the incubation virus period is fourteen days, occurring between four and six days, however there isn't consensus with a view incubation period can be until twenty- four days [4].

The infected individual's proportion by SARS-COV-2 that keep asymptomatic throughout the infection course, aren't clear. In symptomatic patients the clinical manifestations disease, usually start after less than a week, consisting in fever, cough, nasal congestion, weariness and other respiratory signals, infections superior tract.

Gastrointestinal symptoms also appear as nausea or vomiting. Asymptomatic infections were reported in children [5]. Comorbidities presence such as arterial hypertension and lung chronic obstructive disease, also reported as a significant portion in infected patients. The infection can progress to several diseases with dyspnea and severe chest symptoms, corresponding to pneumonia 
especially in elderly patients. Unusual radiographic findings were observed for computered tomography in hospital admission [6] There is a literature consensus [7], explains that the most part of symptomatic individuals shows fever with the complication presence, like cough and dyspnea. Until the moment, we have few studies that describe extrapulmonary systems. However, with the advance cases, become possible and necessary that symptomatic approaches can be studied and described to the community, as Pan studies et al., (2020) that describes the most usual digestive symptom with COVID-19 individuals and [8] that checks diarrhea presence, anorexia and vomit, as initial symptoms infection. Before the exposed, this study aims investigate reports and the patients results with COVID-19 with digestive symptoms.

\section{Methods}

This study is a report case from a COVID-19 patient and gastrointestinal predominantly symptoms. The data were obtained through anamnesis collection contained in the medical report in addition to laboratory tests. Computed tomography of the chest, including RT-PCR to the diagnosis COVID-19.

\section{Case Report}

Patient, F.A.B.M., female, 59 years old, white, married, nurse, born in Rio de Janeiro, sought care due to the complaint of fever and diarrhea. Reports that she started abruptly with fever $\left(38,0^{\circ} \mathrm{C}\right)$, chills and diarrhea of foul-smelling, explosive and with softened consistency. She reports that she used antipyretic during the febrile period and that it lasted for a maximum period of 24 hours after its appearance. It also states that in her work environment there were four cases of positive COVID-19. She denies previous comorbidities, mainly digestive diseases and regular use of medications.

On physical examination, vital signs (SP02: 99\%, RR: 17 breaths / min HR: 88 bpm and blood pressure: 122x80 $\mathrm{mmHg}$ ), regular heart rhythm in 2 times with normal heart sounds without murmur, universally audible vesicular murmur without adventitious noises, abdomen: flaccid and painless on palpation, no signs of peritoneal irritation and no other abnormalities. A diagnostic hypothesis of COVID-19 was suggested.

The patient was instructed by the physician of the clinic where she works to perform the RT-PCR examination for COVID-19 (which aims to detect the genetic material of the virus on average up to the tenth day of symptoms in the patient's sample, thus becoming the "gold standard" methodology in the identification of SARSCoV-2), computed tomography of the chest (which although its findings are nonspecific, similar to other viral infections, plays an important role in evaluating whether there was any degree of pulmonary involvement due to viral infection, such as opacities with attenuation in focal or multifocal, and bilateral ground glass occurring in $50-70 \%$ of cases), probiotic started $12 / 12 \mathrm{~h}$ for 5 days, antipyretic in case of recurrence of febrile condition and remaining in social isolation.
On the fourth day of disease evolution, she presented a significant improvement in the clinical picture, already asymptomatic, obtained the result of Swab that was positive for the new coronavirus and computed tomography of the chest [Figures A \& B] which did not demonstrate any pleuropulmonary involvement characteristic of SARS-CoV-2. After confirmation of COVID-19, it was prescribed Azithromycin 500mg + Ivermectin 6mg, remaining in social isolation and checking in the home environment its saturation that remained normal (98-99\%) throughout the course of the infectious condition.

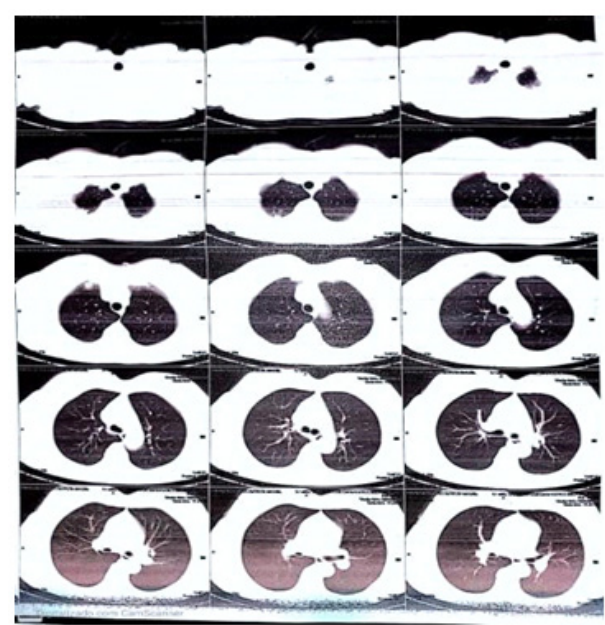

Figure A

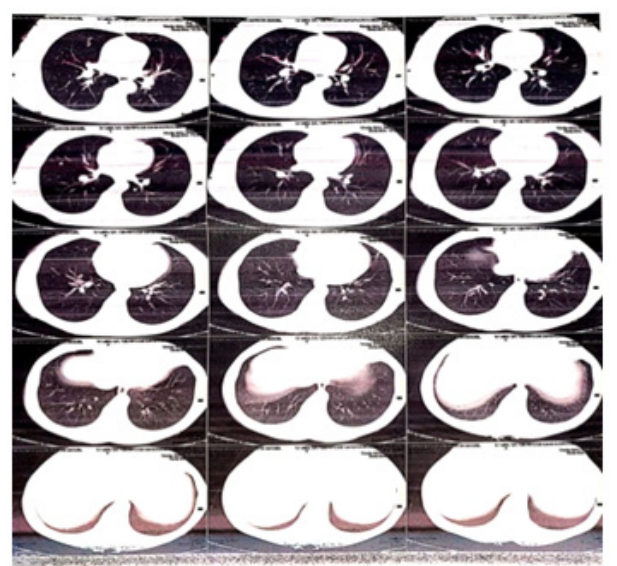

Figure B

Figure A \& B: Chest tomography does not present pleuropulmonary involvement characteristic of SARS-CoV-2.

Fifteen days after the confirmation of the new coronavirus, the patient was instructed to perform serology for COVID-19 in order to detect the presence of IgG class antibodies to define acquired immunity. During the treatment, the patient obtained a satisfactory evolution, without intercurrences and side effect. In the serology result, she presented the presence of IgG class antibodies that indicates that this patient probably developed an immunity against the disease, however, we still do not know what degree of protection is conferred by IgG against SARS-CoV-2 and whether this immunity will be permanent or not. 


\section{Discussion}

The aim of this study identifies the prevalence characteristics or digestive extrapulmonary symptoms, that helped to bring attention to clear the COVID-19 early recognition and to offer treatment faster, before mild illness gets worse. Until the moment, May 2020, pandemic following severity and expansion in all national territory and occurs in other countries yes. The clinical practice shows the symptoms pulmonary prevalence's and breathiness, but there are new identification symptoms, especially in digestive tract. Collaborating with some studies, $[9,10]$ (HUET et al., 2020), show gastrointestinal symptoms, as diarrhea, nauseous and vomit the mainlines. It is important to note, that in critically ill patients, appear many dysfunction organs [11].

It was possible to observe in this study that gastrointestinal symptoms as diarrhea in the beginning was considered unusual in COVID-19, but there is an emerging evidence that is more usual we could think [12].

The retrospective analyses developed by [13] in China, confirmed sample cases, where $88 \%$ show fever, $67,8 \%$ cough,38,1\% weakness, a sputum presence was observed in 33,4 , breathless in $18,6 \%$ report, sore throat in $13,9 \%$ and $13,6 \%$. So, when the gastrointestinal were analyzed, we saw, that $3,8 \%$ had diarrhea, 5\% vomit the collaborated this report case.

There aren't specifically studies about SARS-COV-2 has gastrointestinal symptoms, it is important to remember this virus is similar SARS-COV, so it's possible to enter in human body through angiotensin converting enzyme (ACE-2) which allows the liver tissue lesion through positive regulation expression by ECA-2 in the hepatic organ imported by proliferation hepatocytes originated by cells from biliary duct [8].

Another fact that contributes to this guide, is the inflammatory response caused by SARS-COV can damage the digestive system. Research's $[14,15]$ shows nucleic viral acid presence in stool sample in $53,4 \%$ infected patients. According to observe the intestinal mucous membrane, is virus liable and can bring gastrointestinal symptoms, however, new studies can improve the scientific debate.

Furthermore, we know that intestinal flora is produced by the intestine in a surprising way and different while has been the most important ever. Combined to human body performance, contributing to the nutritional positively metabolism, to develop the maturity of the immunological system and antibacterial effects [16].

There are reports [2,17] infection caused by SARS-COV-2 can damage the intestinal flora, resulting in digestive symptomatic. In this area, the researchers are still taking stool samples to check the intestine and the intestinal flora diversification.
The most important, is highlight which changes and modifications in the function's flora and composition can damage respiratory tract through the immune regulation "referred to lung intestinal axis" which could contribute to identify in these studies that COVID-19 pneumonia patients show symptoms about digestive problems.

In [18] there are variations in hepatic function with COVID-19 patients. According to researchers found gastrointestinal symptoms cause more probability to show hepatic changed tests as AST and ALT when compared to gastric symptom patients.

Finally, studies Pan et al. (2020) noted that, in illness patients can show only gastrointestinal problems. The authors explained the results obtained with the report case, found here [19]. They also report that, symptomatic digestive delayed more than being submitted to exams or tests, extended coagulation and hepatic enzyme. This late is also checking in admission clinical and all these elements [20], difficulty new studies. Therefore, is necessary to analyze the prevalence, evidence, predictors and gastrointestinal symptoms results $[21,22]$.

\section{Conclusions}

The case reported and publications selected bring to light the discussion of the digestive symptoms of COVID-19. The results of the report suggest that physicians should keep in mind that digestive symptoms, such as diarrhea mainly, may be one of the presentation resources of COVID-19; in some cases, they may arise before respiratory symptoms and on rare occasions, it is the only symptom of COVID-19. Physicians should increase their index of suspicion of patients who have digestive symptoms, even in the absence of respiratory symptoms. This knowledge can help with identification of COVID-19, shorter treatment time, earlier quarantine and less exposure to other people.

\section{Reference}

1. Wu F, Zhao S, Yu B, Chen Y, Wang W, et al. (2020) A new coronavirus associated with human respiratory disease in China. Nature 579(7798): 265-269.

2. Zhou P, Yang X, Wang X, Hu B, Zhang L, et al. (2020) A pneumonia outbreak associated with a new coronavirus of probable bat origin. Nature 579(7798): 270-273.

3. Velavan TP, Meyer CG (2020) The COVID-19 epidemic. Trop Med Int Health 25(3): 278-280.

4. Bai Y, Yao L, Wei T, Tian F, Jin D, et al. (2020) Presumed asymptomatic carrier transmission of COVID-19. JAMA 323(14): 1406-1407.

5. Kurtis F Budden, Shaan L Gellatly, David L A Wood, Matthew A Cooper , Mark Morrison, et al. (2017) Emerging pathogenic links between microbiota and the gut-lung axis. Nat Rev Microbiol 15(1): 55-63.

6. Guan GW, Gao L, Wang JW, Wen XJ, Mao TH, et al. (2020) Exploring the mechanism of liver enzyme abnormalities in patients with novel coronavirus-infected pneumonia. Zhonghua Gan Zang Bing Za Zhi 28(2): 100-106. 
7. Guan W, Ni Z, Hu Y, Liang W, Ou C, et al. (2020) Clinical characteristics of 2019 novel coronavirus infection in China. N Engl J Med 382: 17081720 .

8. Gao QY, Chen YX, Fang JY (2020) 2019 Novel coronavirus infection and gastrointestinal tract. J Dig Dis 21(3): 125-126.

9. Chu Hin, Chan Jasper Fuk woo, Yuen Terrence Tsz tai, Shuai Huiping, Yuan Shuofeng, et al. (2020) Comparative tropism, replication kinetics, and cell damage profiling of SARS-CoV-2 and SARS-CoV with implications for clinical manifestations, transmissibility, and laboratory studies of COVID-19: an observational study. The Lancet Microbe 1(1): 14-23.

10. N Yao, SN Wang, JQ Lian, YT Sun, GF Zhang, et al. (2020) Clinical characteristics and influencing factors of patients with novel coronavirus pneumonia combined with liver injury in Shaanxi region [in Chinese] Zhonghua Gan Zang Bing Za Zhi 28(3): 234-239.

11. Xiaobo Yang, Yuan Yu, Jiqian $\mathrm{Xu}$, Huaqing Shu, Yongran $\mathrm{Wu}$, et al (2020) Clinical course and outcomes of critically ill patients with SARSCoV-2 pneumonia in Wuhan, China: A single centered, retrospective, observational study. Lancet Respir Med 8(5): 475-481.

12. GU J, Han B, Wang J (2020) COVID-19: gastrointestinal manifestations and potential fecal-oral transmission. Gastroenterology 158(6): 15181519.

13. Zhang G, Zhang J, Wang B, Zhu X, Wang Q, et al. (2020) Analysis of clinical characteristics and laboratory findings of 95 cases of 2019 novel coronavirus pneumonia in Wuhan, China: a retrospective analysis. Respir Res 21(1): 74

14. An Tang, Zhen Dong Tong, Hong Ling Wang, Ya Xin Dai, Ke Feng Li, et al. (2020) Detection of novel coronavirus by RT PCR in stool specimen from asymptomatic child, China. Emerg Infect Dis 26(6): 1337-1339.

15. Fei Xiao, Meiwen Tang, Xiaobin Zheng, Ye Liu, Xiaofeng Li, et al. (2020) Evidence for gastrointestinal infection of SARS-CoV-2. Gastroenterology 158(6): 1831-1833.

16. Min Li, Baohong Wang, Menghui Zhang, Mattias Rantalainen, Shengyue Wang, et al. (2008) Symbiotic gut microbes modulate human metabolic phenotypes. Proc Natl Acad Sci USA 105(6): 2117-2122.

17. Yang He, Qu Wen, Fangfang Yao, Dong Xu, Yuancheng Huang, et al. (2017) Gut-lung axis: The microbial contributions and clinical implications. Crit Rev Microbiol 43(1): 81-95.

18. Jian Wu, Jun Liu, Xinguo Zhao, Chengyuan Liu, Wei Wang, et al. (2020) Clinical characteristics of imported cases of COVID-19 in Jiangsu province: A multicenter descriptive study. Clin Infect Dis.

19. Chan J, Yuan S, Kok K, To KK, Chu H, et al. (2020) A familial cluster of pneumonia associated with the 2019 novel coronavirus indicating person-to-person transmission: a study of a family cluster. The Lancet 395(10223): 514-523.

20. Hu LL, Wang WJ, Zhu QJ, Yang L (2020) Novel coronavirus pneumonia related liver injury: Etiological analysis and treatment strategy. Zhonghua Gan Zang Bing Za Zhi 28(2): 97-99.

21. Lei Pan, Mi Mu, Pengcheng Yang, Yu Sun, Runsheng Wang, et al. (2020) Clinical Characteristics of COVID-19 Patients With Digestive Symptoms in Hubei, China. Am J Gastroenterol 115(5): 766-773.

22. Dawei Wang, Bo Hu, Chang Hu, Fangfang Zhu, Xing Liu, et al. (2020) Clinical characteristics of 138 hospitalized patients with 2019 novel coronavirus-infected pneumonia in Wuhan, China. JAMA 323(11): 10611069 . 